International Journal of Health Sciences
Available online at www.sciencescholar.us
Vol. 5 No. 3, December 2021, pages: 252-259
e-ISSN: 2550-696X, p-ISSN: 2550-6978
https://doi.org/10.53730/ijhs.v5n3.1467

\title{
Oncologic Outcomes of Pelvic Bone Sarcomas Surgical
}

\author{
CrossMark \\ Djamila Sh. Polatova a , Ulugbek F. Islamov ${ }^{b}$, Rasulbek R. Davletov c, Aleksandr V. Savkin d, \\ Muxiddin M. Sharipov ${ }^{\mathrm{e}}$
}

Manuscript submitted: 09 May 2021, Manuscript revised: 27 August 2021, Accepted for publication: 5 September 2021

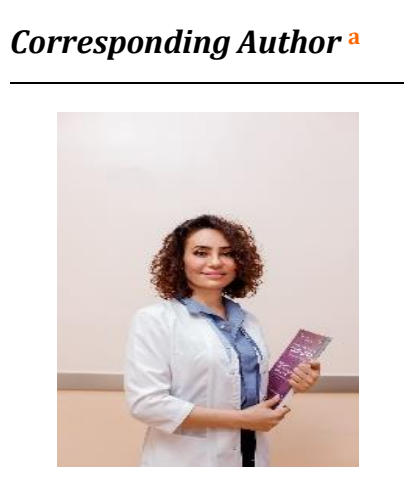

Keywords

oncology;

operations;

pelvic sarcoma;

reconstructive;

survival;

tumor;

\begin{abstract}
Despite the development and implementation of new regimens and methods of adjuvant therapy, one of the complex problems of modern oncology is the treatment of malignant tumors of the pelvic bones, which is reflected in low survival rates and a high percentage of postoperative complications. The article provides a review of the literature on the problem of diagnosis, pathology, and preoperative planning of intraoperative navigation with the definition of the resection zone in pelvic bone sarcomas. Due to the complex anatomical structure of the pelvis, as well as the rare occurrence of pelvic bone sarcomas, a single approach to the treatment of this category of patients has not been developed. The authors conducted a deep analysis of the effectiveness of various types of reconstructive operations from four types of pelvic bone resections, depending on the location of the tumor.
\end{abstract}

International Journal of Health Sciences (C) 2021. This is an open access article under the CC BY-NC-ND license (https://creativecommons.org/licenses/by-nc-nd/4.0/).

\section{Contents}

Abstract

1 Introduction

2 Materials and Methods.

3 Results and Discussions

4 Conclusion

a Republican Specialized Scientific Practical Medical Center of Oncology and Radiology, Tashkent, Republic of Uzbekistan

${ }^{b}$ Republican Specialized Scientific Practical Medical Center of Oncology and Radiology, Tashkent, Republic of Uzbekistan

c Republican Specialized Scientific Practical Medical Center of Oncology and Radiology, Tashkent, Republic of Uzbekistan

d Republican Specialized Scientific Practical Medical Center of Oncology and Radiology, Tashkent, Republic of Uzbekistan

e Republican Specialized Scientific Practical Medical Center of Oncology and Radiology, Tashkent, Republic of Uzbekistan 


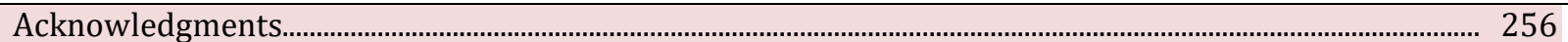

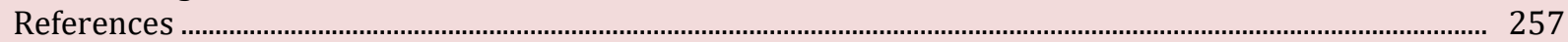

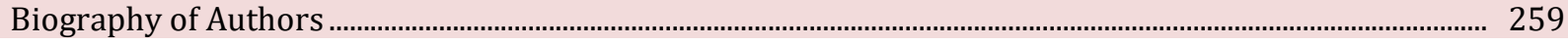

\section{Introduction}

Due to the low prevalence, the variety of histological forms of sarcomas of bones and soft tissues $(0.2-0.6 \%$ of all oncological diseases), their diagnosis and treatment are significant difficulties, little studied and remain the subject of research. Tumor formations of the pelvic bones are characterized by a variety of histological forms, localization of the lesion, clinical course and prognosis. Due to the deep anatomical localization of tumors of the pelvic bones, the clinical manifestation appears late when the tumor is already large. In addition, this area is characterized by a close proximity to the pelvic organs, as well as large blood vessels. Due to the fact that this pathology affects more often people of a young and physically active age, surgical treatment of pelvic tumors should provide both local control over the oncological process and include preservation of the function of the pelvis (Finn, 2012; Lawton et al., 2009).

Most studies have shown that of malignant neoplasms in the pelvic bones, primary bone sarcomas are most common, followed by soft tissue sarcomas and metastatic lesions. When analyzing available sources, inconsistency of data was found regarding the prevalence of various histological types of pelvic sarcomas (Garcia, et al., 2018; Jawad et al., 2011; Wahyudi et al., 2019; Valery et al., 2015). According to studies, pelvic sarcomas account for $10-20 \%$ of bone sarcomas and include histological types such as chondrosarcoma in adults, Ewing's sarcoma in children, and osteosarcoma in adolescents.

According to the most common of the primary pelvic sarcomas is chondrosarcoma, with osteosarcoma in second place. While according to a study by Garcia et al. (2018), osteosarcoma, Ewing's sarcoma, and then chondrosarcomawere more often observed. The reason for the differences is that the number and age of patients varied within different limits, which was reflected in the differences in prevalence rates. The most typical localization of the lesion in pelvic sarcomas is the sciatic tubercle, the iliac-pubic branch, the acetabulum.

In a major research by Stanford University, to study the epidemiological characteristics of patients with tumors of the pelvic bones, as well as their histological characteristics, which included an analysis of 1185 patients with sarcomas of the pelvic bones from 1987 to 2006, the SEER database revealed that the most common chondrosarcoma in 31.3\%, Ewing's sarcoma in 22.1\%, and osteosarcoma in $19 \%$ of cases. In this case, it is necessary to note the difference in the variation in the prevalence of various histological subtypes depending on the age subgroup. In most cases, the tumor is represented by a solitary lesion (97.9\%). In the presented study Jawad et al. (2011), it was noted that pelvic sarcomas have an extremely poor prognosis.

According to various studies, survival rates for pelvic sarcomas vary between 45-55\%. It should be noted that, depending on the histological characteristics of the tumor, survival also varied significantly. For example, the most unfavorable histological type of sarcomas is osteosarcoma, 5 year survival according to various authors is from 4 to $32 \%$. At the same time, 5 -year survival in chondrosarcoma ranged from 72 to $92 \%$. In early studies, the survival rate for Ewing's sarcoma was 20-30\%, while later studies showed a significant improvement in 5-year survival to $46 \%$, which may be due to improved multimodal therapy.

\section{Materials and Methods}

Factors affecting survival, according to Kawai et al. (1998), were the status of the resection margins and the stages of the tumor process, according to Wirbel et al. (2001), tumor size and type of surgery, while the study by Shin et al. the prognostic value of the degree of tumor differentiation has been proven. Other important prognostic parameters include the histological type of tumor and the age of the patient (Laitinen et al., 2015). Resections of the pelvic bones, especially in the area of the sciatic tuber and the sacroiliac joint, are technically difficult surgical interventions, due to the complex geometric three-dimensional structure of the pelvic bones, as well as the proximity of important organs and neurovascular structures. A high percentage of local relapses

Polatova, D. S., Islamov, U. F., Davletov, R. R., Savkin, A. V., \& Sharipov, M. M. (2021). Oncologic outcomes of pelvic bone sarcomas surgical. International Journal of Health Sciences, 5(3), 252-259. https://doi.org/10.53730/ijhs.v5n3.1467 
is due to the complexity of the anatomical and topographic structure of the pelvis and the limited operative field, and according to various authors it is $28-35 \%$. In addition, improper planning, without taking into account the soft tissues of the pelvic region, or its failure, leads to the incorrect implementation of intraoperative osteotomy, which, in turn, can have far-reaching consequences, which are reflected in a decrease in local control (Salunke et al., 2017; Pestieau et al., 2000).

According to a number of studies, only a wide resection of the pelvic bones can achieve low rates of local recurrence, due to the possibility of achieving "clean" resection edges. In this connection, performing surgery on the pelvic bones requires thorough preoperative preparation and a high level of training for the surgeon. It should be noted that even experienced operators during surgical interventions may not achieve adequate resection margins. In support of this, in Cartiaux et al. (2008), an in vitro study, which demonstrated insufficient accuracy of the performed resections in cases of lack of preoperative planning and navigation methods, which was expressed in more frequent detection of positive edges of the resection. In the study, the average distance between the tumor and the safe edges of the resection was $5.3 \mathrm{~mm}$, ranging from 2.7 to 5.3 $\mathrm{mm}$. Even under the condition of surgical intervention by experienced surgeons, there were cases of undergoing a tumor resection incision, which in turn violated the aplastic conditions of the operation.

Therefore, preoperative planning using radiation imaging methods (MRI, CT) allows you to perform the optimal number of organ-sparing operations, the main purpose of which is to increase the accuracy of osteotomy in order to achieve negative margins of resection and prevent local recurrences. In addition, the above methods make it possible to assess the prevalence of the tumor process, as well as the relative position of important structures (nerves, blood vessels, internal organs), as well as conduct their 3D modeling, which helps prevent their intraoperative damage (Bradbury et al., 2006; Chakravarty et al., 2000).

There are works on the study of intraoperative navigation, which allows the use of this technology for resection of bone sarcomas, reaching the negative edges of the resection. A decrease in the incidence of local recurrence was achieved by applying the above technology in (Jeys et al., 2013). Recent studies in the field of 3D modeling and printing models, taking into account the individual anatomical features of the patient, as well as special tools, allow preliminary 3D planning of the operation and adequate reconstruction of the pelvic bones. A recent report by Gouin et al. showed that the accuracy of determining the location of the edges of the upcoming resection averaged 2.5 millimeters $(\mathrm{mm})$ in nine planes of the section and illustrates the different levels of complexity of three-dimensional planning; from conventional osteotomy by hand to models with a PSI print using cutting units (Gouin et al., 2014). The limitation of this study is the small number of patients, as well as the heterogeneity of the histological structure of the sarcomas of the pelvic bones.

\section{Results and Discussions}

Due to the insufficient effectiveness of adjuvant therapy until the early 1980-s, crippling operations were the method of choosing malignant tumors of the pelvic bones (Umer et al., 2017). Carrying out this operation presents significant difficulties due to the large size of the tumors, the complexity of the anatomical structure of the pelvis, as well as diligence or germination in the neurovascular bundle. Currently, according to a study Jemal et al. (2010), the number of hemipelvectomies performed annually is 1 case per 1 million population.

Today, the use of crippling surgery by most researchers is justified, only in case of unrepeatability of the tumor process, in connection with the size or invasion of the internal organs, as well as nerves and blood vessels. With the subsequent development of preoperative imaging methods and modern multimodal treatment regimens, it became possible to use operations with preservation of the lower extremities. The choice of tactics for surgical treatment depends on the histological type, location and prevalence of the tumor process, its position in relation to the acetabulum. The main condition for the use of organ-preserving operations for tumors of the pelvic bones is to achieve negative edges of the resection. Tumor localization affects the stability and function of the pelvic bones, for example, when the tumor is localized in the area of the ilium or pubic bone, the pelvic stability is not violated, while when the tumor is located in the posterior part of the iliac bone, it can affect the integrity of the iliac-sacral joint, and tumors in the acetabulum negatively affect the function of the thigh and the bearing capacity of the lower limb (Zerr et al., 1997; Barratt et al., 2011).

When analyzing the available literature, a small number of studies on the functional results of the surgical treatment of pelvic bone tumors were revealed. Currently, it remains relevant to conduct pelvic resections 
according to the classification of Enneking and Dunham, according to which 4 types of resections are divided depending on the location of the tumor. Pelvic resection of the pelvis I, used for tumor damage to the ilium, often leads to pelvic instability due to the appearance of a pelvic ring defect. Due to the fact that the resection of the ilium without reconstruction leads to instability and a defect in the pelvic ring, this negatively affects its functional state. Therefore, only the use of reconstructive operations for the restoration of the ilium is necessary to maintain the stability of the pelvis and provide mobility. In general, the results of pelvic bone resection of type I are favorable, in addition, in most patients there is a restoration of independent movement. Organ-sparing operations with the location of the tumor in the acetabulum are a complex type of surgical intervention. The results of the treatment of tumors of this localization remain unsatisfactory. Initially, some authors advocated the use of pseudarthrosis and arthrodesis in surgical procedures of type II to maintain function (Glimelius et al., 1996; Michal et aL., 2000).

However, pseudoatrosis during such operations was unstable and painful, and ischiofemoral or ileofemoral arthrodesis the treatment results were unsatisfactory, and also led to a significant discrepancy in the length of the limbs and difficulties with sitting and walking. According to studies on the use of allografts, good treatment results were achieved, however, complications in the form of allograft fractures and infectious complications were observed in this category of patients (Brown et al., 2018; Ogura et al., 2018). To date, the use of saddles Mark II is widely used in reconstructions after resection of the pelvic bones of type II. A number of studies have shown good treatment results, while the number of various complications remains high (53\%) (Ogura et al., 2018; Widana et al., 2021). Although reconstruction using allografts is one of the most common reconstruction options, a study of the results of existing studies shows that it is associated with a high frequency of complications, including fractures, the absence of bone fusion and infection (Brown et al., 2018). 5 cases of pelvic reconstruction using a vascularized iliac auto graft fixed with a foot screw and a shaft that showed bone fusion were reported for the first time (Tsuchiya et al., 2012).

Surgical interventions when the sacroiliac joint is involved in the process are the most complex and highly traumatic surgical interventions for tumors of the pelvic bones due to the extended dissection of the two zones of the pelvic ring, and are characterized by the development of a large number of postoperative complications. According to various authors, in $26-54 \%$ of cases, the edges of the resection remain inadequate, which is reflected in the high rates of development of local relapses. According to a study Sabourin et al. (2009), there were no statistically significant differences in the rates of relapse-free and overall survival with hemisacromectomy and resection of the ileo-sacral joint, provided negative margins of resection were achieved, however, in the group of patients after hemisacromectomy, the functional indices were significantly lower, which indicates preference for the use of organ-sparing operations in the localization of tumors in this area. It should be noted that the results of treatment still remain disappointing, the 5-year survival rate of patients with lesion of the ileo-sacral joint is $50 \%$. According to studies, the rates of development of local relapses vary widely $4-34 \%$.

For the reconstruction of this area, several authors have proposed several reconstruction options such as vascularized and non-vascularized auto grafts, allografts, modular implants. However, the question of the appropriateness of reconstructive operations for tumor localization in the area of the ileo-sacral joint is still a matter of debate. For example, in the work of Beadel et al. (2005), in a comparative analysis of the treatment results, in the group of patients without reconstructive interventions, a lower number of complications, a lesser need for permanent postoperative pain relief for chronic pain syndrome, were compared with the group of patients subjected to reconstruction. While in other studies, such as Delloye et al. (2007); Langlais et al. (2001), it was noted that the use of allografts allows achieving good treatment results, as well as high rates of mechanical strength in this area, which has persisted for a long time. According to various authors, the overall survival of patients after surgical treatment varies from 40 to $70 \%$. According to [20], factors affecting the prognosis of patients with malignant tumors of a given localization include age, degree of differentiation, and tumor volume.

Complications after organ-sparing procedures for pelvic bone tumors develop in about $1 / 3$ of patients, especially those who underwent reconstruction with end prosthetics. According to studies, purulent-septic complications are most common, which is associated with a high invasiveness of operations, the formation of extensive cavities and concomitant resection of soft tissues. In addition, the duration of surgery, the proximity of the tumor to the rectum and urogenital tract, the presence of extensive dead spaces, foreign material contributes to infectious complications, in particular during reconstructive operations of the pelvis. In the

Polatova, D. S., Islamov, U. F., Davletov, R. R., Savkin, A. V., \& Sharipov, M. M. (2021). Oncologic outcomes of pelvic bone sarcomas surgical. International Journal of Health Sciences, 5(3), 252-259. https://doi.org/10.53730/ijhs.v5n3.1467 
study Puchner et al. (2017), showed a low number of postoperative complications and the possibility of early rehabilitation when using pelvic resections with subsequent use of reconstructive interventions.

Available reports indicate high rates of postoperative complications associated with surgical resection and provide conflicting data on treatment outcomes and prognosis. The reasons for this are that most of the existing studies were conducted in only one center (single-center study), in connection with which there was a wide variation in the choice of patients and treatment methods, as well as a small number of observations, which reflected on the statistical reliability of each study, and finally, inconsistency in the analysis of the data and their heterogeneity, for example, in some studies, the results and prognostic factors were analyzed for a particular histopathological subtype of the tumor, while in other studies, various histopathological subtypes of pelvic bone tumors were grouped.

Such heterogeneity of data causes certain difficulties in interpreting the results of studies, and raises a number of questions, such as whether the tactics of managing patients with bone tumors is applicable regardless of the histological type of patients, or whether the approach to treatment should be determined depending on the structure of the tumor. An example of this is the conflicting data on the treatment of Ewing's pelvic sarcoma, when one group of authors insists on the use of radiation therapy as an alternative to surgical intervention, while a number of authors emphasize the importance of broad resection of the primary tumor in all types of sarcomas, including sarcoma Ewing (Dharmayuda et sl., 2021; Kurtieva et al., 2021).

\section{Conclusion}

Thus, the article analyzed the practical experience of using various surgical tactics for resection of the pelvic bones, depending on the localization of the tumor. The localization of the tumor continues to play a very important role in the effectiveness of applicable practices and the occurrence of postoperative complications. Heterogeneity of data causes certain difficulties in interpreting the results of studies, and raises a number of questions, such as whether the tactics of managing patients with bone tumors is applicable regardless of the histological type of patients, or whether the approach to treatment should be determined depending on the structure of the tumor. It was noted that the use of allografts allows achieving good treatment results, as well as high rates of mechanical strength in this area, which has persisted for a long time.

Considering that the main category of patients with such a diagnosis is young people, surgical treatment of pelvic tumors should provide both local control over the oncological process and include preservation of pelvic function. Thus, only a multimodal, personalized approach to the treatment of pelvic bones, taking into account individual and anatomical characteristics, the balance between oncological control and the preservation of functionality and the desire for a better quality of life, can achieve good treatment results for this category of patients. In this connection, it is necessary to improve the surgical tactics of this category of patients in order to reduce the number of complications, as well as the development of rehabilitation measures in the early stages.

\section{Acknowledgments}

We are grateful to two anonymous reviewers for their valuable comments on the earlier version of this paper. 


\section{References}

Barratt, M., Choi, T. Y., \& Li, M. (2011). Qualitative case studies in operations management: Trends, research outcomes, and future research implications. Journal of operations management, 29(4), 329-342. https://doi.org/10.1016/j.jom.2010.06.002

Beadel, G. P., McLaughlin, C. E., Wunder, J. S., Griffin, A. M., Ferguson, P. C., \& Bell, R. S. (2005). Outcome in two groups of patients with allograft-prosthetic reconstruction of pelvic tumor defects. Clinical Orthopaedics and Related Research (1976-2007), 438, 30-35.

Bradbury, E. T., Simons, W., \& Sanders, R. (2006). Psychological and social factors in reconstructive surgery for hemi-facial palsy. Journal of Plastic, Reconstructive \& Aesthetic Surgery, 59(3), 272-278. https://doi.org/10.1016/j.bjps.2005.09.003

Brown, T. S., Salib, C. G., Rose, P. S., Sim, F. H., Lewallen, D. G., \& Abdel, M. P. (2018). Reconstruction of the hip after resection of periacetabular oncological lesions: a systematic review. The bone \& joint journal, 100(1_Supple_A), 22-30.

Cartiaux, O., Docquier, P. L., Paul, L., Francq, B. G., Cornu, O. H., Delloye, C., ... \& Banse, X. (2008). Surgical inaccuracy of tumor resection and reconstruction within the pelvis: an experimental study. Acta orthopaedica, 79(5), 695-702.

Chakravarty, B., Konar, H., \& Chowdhury, N. N. R. (2000). Pregnancies after reconstructive surgery for congenital cervicovaginal atresia. American journal of obstetrics and gynecology, 183(2), 421-423. https://doi.org/10.1067/mob.2000.105740

Delloye, C., Banse, X., Brichard, B., Docquier, P. L., \& Cornu, O. (2007). Pelvic reconstruction with a structural pelvic allograft after resection of a malignant bone tumor. JBJS, 89(3), 579-587.

Dharmayuda, T. G., Suega, K., Bakta, I. M., \& Sumohadi, I. M. D. (2021). Ki67 expression and prognostic aspects of colorectal cancer. International Journal of Health Sciences, 5(2), 79-88. https://doi.org/10.29332/ijhs.v5n2.1215

Finn, O. J. (2012). Immuno-oncology: understanding the function and dysfunction of the immune system in cancer. Annals of oncology, 23, viii6-viii9. https://doi.org/10.1093/annonc/mds256

Garcia, J. G., Martinez, A., Garcia, R. J., Petrilli, M. T., \& Viola, D. C. (2018). Epidemiological characteristics of patients with pelvic tumors submitted to surgical treatment 2 . Revista brasileira de ortopedia, 53, 33-37.

Glimelius, B., Hoffman, K., Sjödén, P. O., Jacobsson, G., Sellström, H., Enander, L. K., ... \& Svensson, C. (1996). Chemotherapy improves survival and quality of life in advanced pancreatic and biliary cancer. Annals of oncology, 7(6), 593-600. https://doi.org/10.1093/oxfordjournals.annonc.a010676

Gouin, F., Paul, L., Odri, G. A., \& Cartiaux, O. (2014). Computer-assisted planning and patient-specific instruments for bone tumor resection within the pelvis: a series of 11 patients. Sarcoma, 2014.

Jawad, M. U., Haleem, A. A., \& Scully, S. P. (2011). Malignant sarcoma of the pelvic bones: treatment outcomes and prognostic factors vary by histopathology. Cancer, 117(7), 1529-1541.

Jemal, A., Siegel, R., Xu, J., \& Ward, E. (2010). Cancer statistics, 2010. CA: a cancer journal for clinicians, 60(5), 277-300.

Jeys, L., Matharu, G. S., Nandra, R. S., \& Grimer, R. J. (2013). Can computer navigation-assisted surgery reduce the risk of an intralesional margin and reduce the rate of local recurrence in patients with a tumour of the pelvis or sacrum?. The bone \& joint journal, 95(10), 1417-1424.

Kawai, A., Huvos, A. G., Meyers, P. A., \& Healey, J. H. (1998). Osteosarcoma of the pelvis. Oncologic results of 40 patients. Clinical orthopaedics and related research, (348), 196-207.

Kurtieva, S., Nazarova, J., \& Mullajonov, H. (2021). Features of endocrine and immune status in adolescents with vegetative dystonia syndrome. International Journal of Health Sciences, 5(2), 118-127. https://doi.org/10.29332/ijhs.v5n2.1332

Laitinen, M., Parry, M., Albergo, J. I., Jeys, L., Abudu, A., Carter, S., ... \& Grimer, R. (2015). The prognostic and therapeutic factors which influence the oncological outcome of parosteal osteosarcoma. The bone \& joint journal, 97(12), 1698-1703.

Langlais, F., Lambotte, J. C., \& Thomazeau, H. (2001). Long-term results of hemipelvis reconstruction with allografts. Clinical Orthopaedics and Related Research ${ }^{\circledR}, 388,178-186$.

Lawton, C. A., Michalski, J., El-Naqa, I., Buyyounouski, M. K., Lee, W. R., Menard, C., ... \& Seider, M. (2009). RTOG GU Radiation oncology specialists reach consensus on pelvic lymph node volumes for high-risk prostate

Polatova, D. S., Islamov, U. F., Davletov, R. R., Savkin, A. V., \& Sharipov, M. M. (2021). Oncologic outcomes of pelvic bone sarcomas surgical. International Journal of Health Sciences, 5(3), 252-259.

https://doi.org/10.53730/ijhs.v5n3.1467 
cancer. International Journal of Radiation Oncology* Biology* Physics, 74(2), 383-387. https://doi.org/10.1016/j.ijrobp.2008.08.002

Michal, M., Hes, O., \& Havlicek, F. (2000). Benign renal angiomyoadenomatous tumor: a previously unreported renal tumor. Annals of diagnostic pathology, 4(5), 311-315. https://doi.org/10.1053/adpa.2000.17890

Ogura, K., Susa, M., Morioka, H., Matsumine, A., Ishii, T., Hamada, K., ... \& Kawai, A. (2018). Reconstruction using a constrained-type hip tumor prosthesis after resection of malignant periacetabular tumors: A study by the Japanese Musculoskeletal Oncology Group (JMOG). Journal of surgical oncology, 117(7), 1455-1463.

Pestieau, S. R., Jelinek, J. S., Chang, D., Jacquet, P., \& Sugarbaker, P. H. (2000). CT in the selection of patients with abdominal or pelvic sarcoma for reoperative surgery. Journal of the American College of Surgeons, 190(6), 700-710. https://doi.org/10.1016/S1072-7515(00)00253-2

Puchner, S. E., Funovics, P. T., Böhler, C., Kaider, A., Stihsen, C., Hobusch, G. M., ... \& Windhager, R. (2017). Oncological and surgical outcome after treatment of pelvic sarcomas. PloS one, 12(2), e0172203.

Sabourin, M., Biau, D., Babinet, A., Dumaine, V., Tomeno, B., \& Anract, P. (2009). Surgical management of pelvic primary bone tumors involving the sacroiliac joint. Orthopaedics \& Traumatology: Surgery \& Research, 95(4), 284-292.

Salunke, A. A., Shah, J., Warikoo, V., Chakraborty, A., Sahijwani, H., Sharma, M., ... \& Jain, A. (2017). Surgical management of pelvic bone sarcoma with internal hemipelvectomy: Oncologic and Functional outcomes. Journal of clinical orthopaedics and trauma, 8(3), 249-253. https://doi.org/10.1016/j.jcot.2017.04.004

Tsuchiya, H., Shirai, T., Nishida, H., Murakami, H., Kabata, T., Yamamoto, N., ... \& Nakase, J. (2012). Innovative antimicrobial coating of titanium implants with iodine. Journal of Orthopaedic Science, 17(5), 595-604.

Umer, M., Ali, M., Rashid, R. H., Mohib, Y., \& Rashid, H. U. (2017). Outcomes of internal hemipelvectomy for pelvic tumors: a developing country's prospective. International journal of surgery. Oncology, 2(4), e07.

Valery, P. C., Laversanne, M., \& Bray, F. (2015). Bone cancer incidence by morphological subtype: a global assessment. Cancer Causes \& Control, 26(8), 1127-1139.

Wahyudi, M., Kamal, A. F., \& Prabowo, Y. (2019). Outcomes for pelvic tumors treated by pelvic resection. Case series. International Journal of Surgery Open.

Widana, I.K., Sumetri, N.W., Sutapa, I.K., Suryasa, W. (2021). Anthropometric measures for better cardiovascular and musculoskeletal health. Computer Applications in Engineering Education, 29(3), 550561. https://doi.org/10.1002/cae.22202

Wirbel, R. J., Schulte, M., \& Mutschler, W. E. (2001). Surgical treatment of pelvic sarcomas: oncologic and functional outcome. Clinical Orthopaedics and Related Research (1976-2007), 390, 190-205.

Zerr, K. J., Furnary, A. P., Grunkemeier, G. L., Bookin, S., Kanhere, V., \& Starr, A. (1997). Glucose control lowers the risk of wound infection in diabetics after open heart operations. The Annals of thoracic surgery, 63(2), 356-361. https://doi.org/10.1016/S0003-4975(96)01044-2 


\section{Biography of Authors}

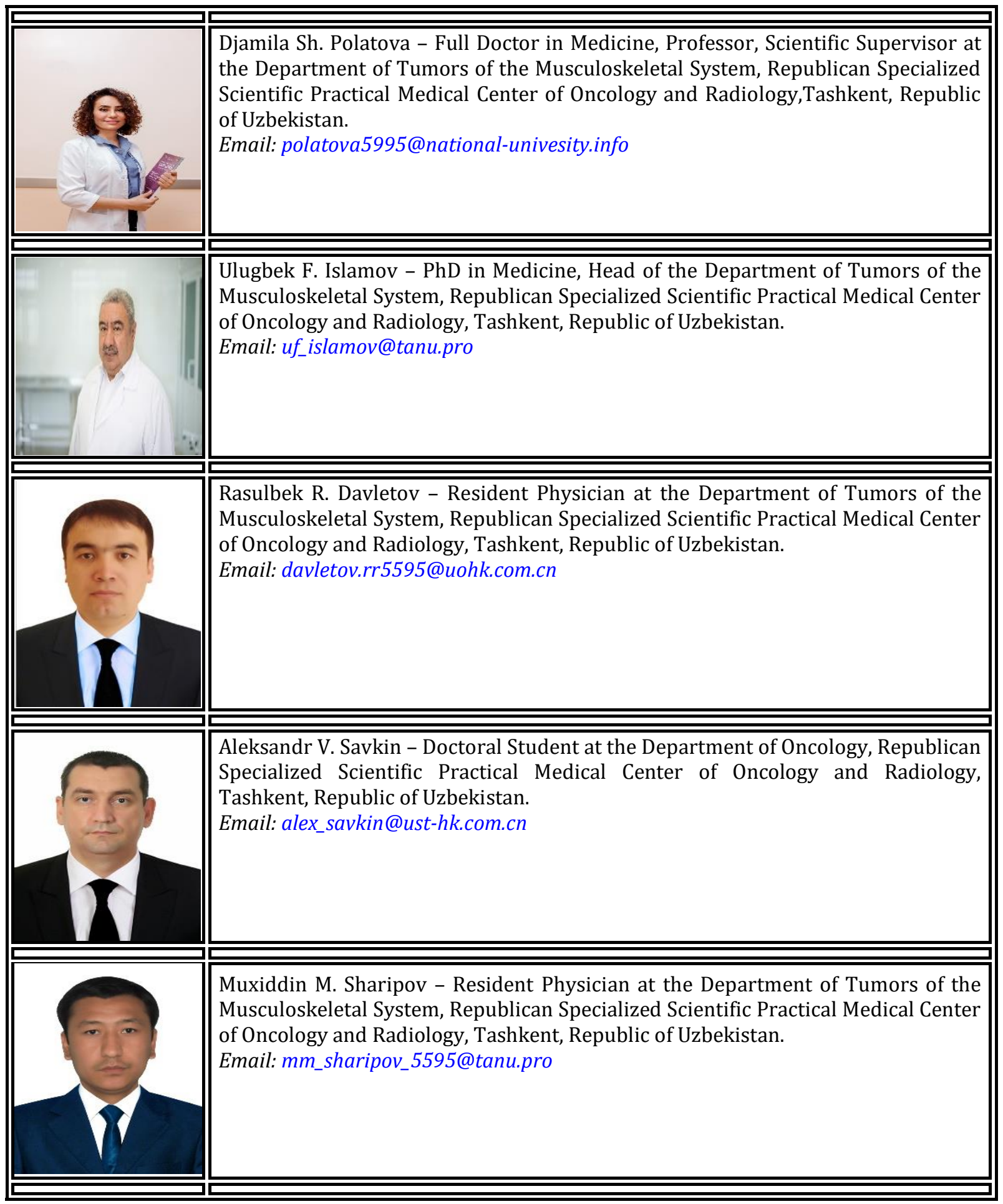

Polatova, D. S., Islamov, U. F., Davletov, R. R., Savkin, A. V., \& Sharipov, M. M. (2021). Oncologic outcomes of pelvic bone sarcomas surgical. International Journal of Health Sciences, 5(3), 252-259. https://doi.org/10.53730/ijhs.v5n3.1467 